\title{
Análisis espacial-temporal de procesos relacionados con concentraciones de oxígeno disuelto en lagunas de maduración
}

María B. Arévalo1, Wout Van Echelpoel ${ }^{2}$ (D), Andrés O. Alvarado ${ }^{3,4}$, Peter L. M. Goethals ${ }^{2}$, Josué B. Larriva ${ }^{1,5}$

${ }^{1}$ Facultad de Ciencia y Tecnología, Universidad del Azuay, Av. 24 de Mayo 7-77 y Hernán Malo, Cuenca, Ecuador.

2 Aquatic Ecology Research Unit, Department of Applied Ecology and Environmental Biology, Ghent University, Coupure Links 653, B-9000 Ghent, Belgium.

${ }^{3}$ Departamento de Recursos Hídricos y Ciencias Ambientales, Universidad de Cuenca, Av. 12 de Abril, Cuenca, Ecuador.

${ }^{4}$ Facultad de Ingeniería, Universidad de Cuenca, Av. 12 de Abril, Cuenca, Ecuador.

${ }^{5}$ Empresa Pública Municipal de Telecomunicaciones, Agua Potable, Alcantarillado y Saneamiento de Cuenca (ETAPA EP).

Autor para correspondencia: barevalo@uazuay.edu.ec

Fecha de recepción: 5 de octubre 2017 - Fecha de aceptación: 28 de noviembre 2017

\section{RESUMEN}

El artículo presenta un análisis de la variabilidad de concentraciones de oxígeno disuelto en el tiempo y espacio en las lagunas de maduración de la Planta de Tratamiento de Aguas Residuales de Ucubamba (Cuenca, Ecuador), en función de diferentes parámetros físicos, químicos, biológicos y meteorológicos. Se utilizó un enfoque estadístico mediante modelos de efectos mixtos para evaluar la variabilidad de las concentraciones de oxígeno disuelto en forma espacial y temporal en cada laguna y analizar la influencia de los factores meteorológicos, químicos y biológicos, sobre las concentraciones de oxígeno disuelto. La profundidad afectó significativamente las concentraciones de oxígeno disuelto, y de igual manera, el fósforo, según la hora del día. Por otro lado, el pH solo mostró efecto en una de las dos lagunas analizadas. Además, la clorofila $a$, como indicador de la concentración de la biomasa de algas, tuvo una correlación positiva con el oxígeno disuelto, siguiendo los dos parámetros tendencias similares, i.e. con mayores concentraciones cerca de la superficie. Los factores meteorológicos no mostraron ningún efecto sobre las concentraciones de oxígeno disuelto.

Palabras clave: Tratamiento de agua residual, estadística, oxígeno disuelto, Ecuador.

\begin{abstract}
This article presents the analysis of the variability of dissolved oxygen concentrations in time and space along the maturation ponds of the wastewater treatment plant of Ucubamba (Cuenca, Ecuador), according to physical, chemical and biological parameters, and meteorological conditions. A statistical approach with mixed effects models was used for testing the influence of the different variables through space and time, on dissolved oxygen concentrations. Depth was the most significant factor affecting dissolved oxygen. Phosphorous in the maturation ponds, depending on the hour of the day, presented an effect on dissolved oxygen concentrations. $\mathrm{pH}$, on the other hand, only showed significance in one of the two ponds analyzed. Further, chlorophyll $a$, as a proxy for algal biomass concentration, had a positive correlation with dissolved oxygen following similar trends with higher concentrations closer to the surface. Meteorological factors did not show any significant effect on dissolved oxygen.
\end{abstract}

Keywords: water treatment, statistics, dissolved oxygen, Ecuador. 


\section{INTRODUCCIÓN}

El correcto tratamiento del agua residual es un asunto de suma importancia tanto para el medio ambiente como para la salud pública. Sin embargo, en Ecuador, más del 90\% de las aguas residuales domésticas son descargadas a un cuerpo receptor sin recibir ningún tratamiento previo (Alvarado, 2013). La ciudad de Cuenca, situada en el sur de Ecuador, cuenta con una de las pocas plantas de tratamiento de aguas residuales (PTAR) que existen en el país. Esta planta consiste en un sistema de lagunas de estabilización: dos líneas paralelas de lagunas aireadas, facultativas y maduración, en serie.

Las lagunas de estabilización son una alternativa muy eficiente de tratamiento a bajo costo, confiable y sostenible en el tiempo (Mburu, Tebitendwa, van Bruggen, Rousseau, \& Lens, 2013), que además posee altas eficiencias para la disminución de materia orgánica y patógenos (Mahmood, Pervez, Zeb, Zaffar, Yaqoob et al., 2013). Estos últimos se remueven mayoritariamente en las lagunas de maduración, que representan la última etapa del tratamiento. Diferentes mecanismos ocurren en dichas lagunas para la remoción de los patógenos, los cuales se encuentran directamente relacionados con la radiación solar, sedimentación, biodegradación, predación, respiración endógena, pH y concentraciones de oxígeno disuelto (OD) (Rivas, Barceló-Quintal, \& Moeller, 2011; Von Sperling, 2007; Dias, Passos, \& Von Sperling, 2017). Liu et al. (2015) encontró que con altos valores de $\mathrm{pH}$ y oxígeno disuelto (OD), ocurre una desinfección efectiva, es así que, las variaciones de OD en lagunas de maduración podrían dar una percepción del estado de remoción de patógenos. A su vez, los niveles de OD dependen de diferentes procesos de consumo y producción, que incluyen parámetros físicos, químicos y biológicos (Walmsley \& Shilton, 2005).

Con el objetivo de conocer apropiadamente los procesos relacionados con el OD se debe tomar en cuenta los parámetros individuales que tengan influencia sobre él y sus cambios en el tiempo y espacio. Los modelos estadísticos representan una herramienta útil para analizar esa clase de variaciones y relaciones (Adèr, Mellenbergh, \& Hand, 2008). En este trabajo se utiliza un modelo linear de efectos mixtos para probar la influencia de parámetros físico, químicos, biológicos y factores meteorológicos sobre el OD en el tiempo y espacio en lagunas de maduración.

\section{MATERIALES Y MÉTODOS}

\subsection{El sistema de lagunas de estabilización}

Las muestras fueron tomadas en las lagunas de maduración de la PTAR de Ucubamba. Esta planta trata el efluente proveniente del alcantarillado combinado de Cuenca (caudal medio $1.2 \mathrm{~m}^{3} \mathrm{~s}^{-1}$ ), una ciudad con 505,585 habitantes, situada a 2,560 m.s.n.m al sur del Ecuador y con temperatura media anual de $14^{\circ} \mathrm{C}$.

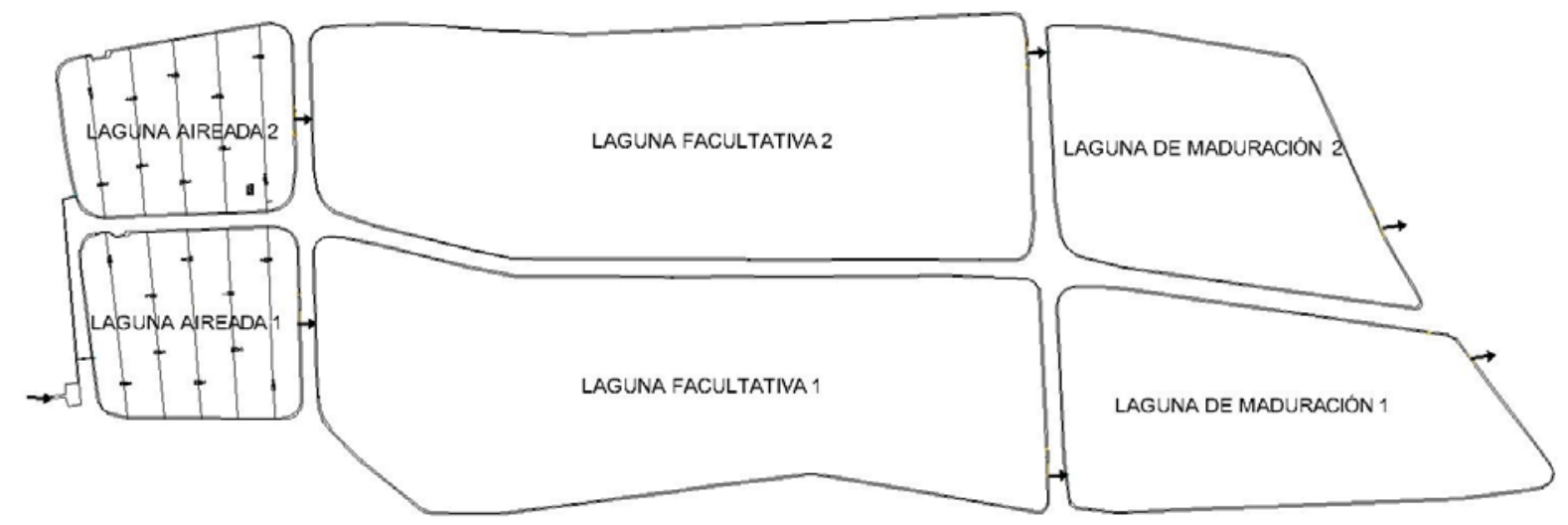

Figura 1. Sistema de lagunas de estabilización de la Planta de Tratamiento de Aguas Residuales de Ucubamba. 
Las lagunas de maduración son el último paso en el tratamiento biológico. Estas lagunas ocupan un área de 13 ha (7.4 ha para la laguna de maduración en la primera línea y 5.6 ha para la laguna de la segunda línea), con una profundidad de $2 \mathrm{~m}$. El influente de estas lagunas corresponde al efluente de las lagunas facultativas (Fig. 1). El período de retención hidráulico para la laguna de maduración en la línea 1 (M1) es de 2.9 días y para la laguna de maduración en la línea 2 (M2) es de 2.2 días.

\subsection{Localización de los puntos de muestreo}

Se colocaron 9 puntos de muestreo en cada laguna, como se observa en la Fig. 2. Se recogieron dos muestras en cada punto, una a $0.3 \mathrm{~m}$ debajo del nivel del agua (superficie) y otra a $0.15 \mathrm{~m}$ sobre el sedimento (fondo). Se llevaron a cabo tres campañas de muestreo durante el 25 y 26 de julio (T1), 14 y 15 de agosto (T2), y 26 y 27 de agosto (T3) de 2013.

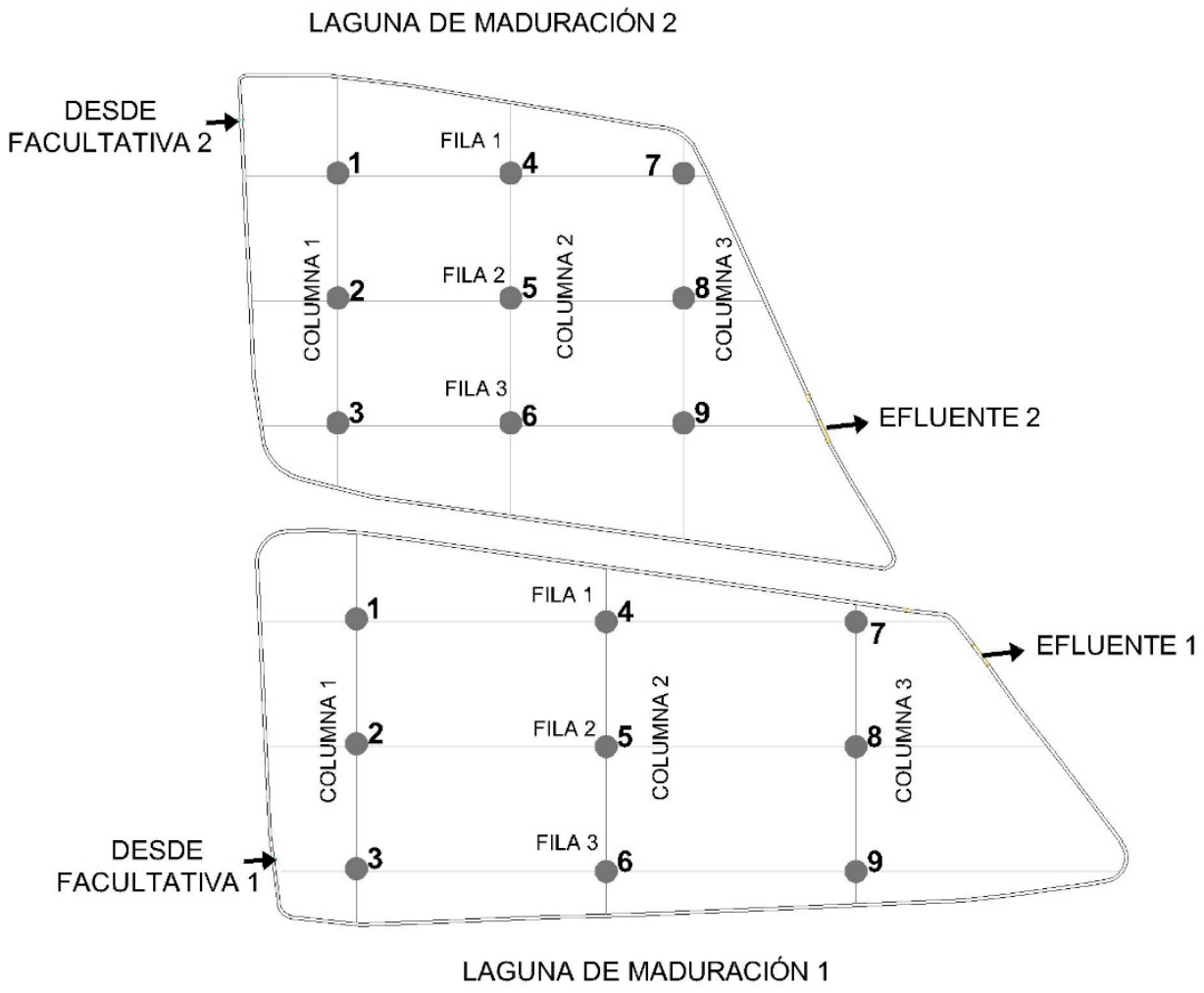

Figura 2. Localización de puntos de muestreo.

\subsection{Parámetros físico químicos y biológicos}

Los parámetros físico, químicos y biológicos se muestrearon de dos maneras, para ser analizados en el laboratorio o medidos directamente con una multi-probe. Se utilizaron dos multi-probes manuales, YSI 6600 V2 y YSI 6920 V1, para monitorear pH, clorofila $a$, y OD in situ. Al mismo tiempo que se midieron los parámetros in situ, se tomaron muestras de $300 \mathrm{~mL}$ con un muestreador automático (Teledyne ISCO, modelo 6712). Las muestras tomadas en cada punto de cada columna (Fig. 2) fueron mezcladas, resultando 3 muestras para cada profundidad en cada laguna, de $900 \mathrm{~mL}$. Se obtuvo un total de 36 muestras (6 muestras por laguna para cada campaña), las cuales se analizaron de acuerdo a "Standard Methods for Examination of Water and Wastewater" (APHA, 2005) para $\mathrm{DBO}_{5}$, DQO, P y N Kjeldahl.

\subsection{Datos meteorológicos}

La temperatura media del aire $\left({ }^{\circ} \mathrm{C}\right)$, radiación solar $\left(\mathrm{W} \mathrm{m}^{-2}\right)$, velocidad del viento $\left(\mathrm{m} \mathrm{s}^{-1}\right)$ y precipitación (mm) se obtuvieron de la Estación Meteorológica de CELEC HidroPaute localizada en las coordenadas -2.859308; -78.933909, aproximadamente a $600 \mathrm{~m}$ de distancia de la PTAR. 


\subsection{Desarrollo del modelo}

Se utilizó el modelo estadístico linear de efectos mixtos para probar la variabilidad de OD en función de los diferentes parámetros físico químicos, biológicos y factores meteorológicos en el espacio y tiempo. El uso de este modelo se justifica debido a la correlación existente entre las mediciones repetidas de OD en los diferentes puntos de las lagunas. Este modelo asume que las concentraciones de OD se encuentran linealmente relacionadas a un grupo de variables explicativas (localización, profundidad, hora, condiciones climáticas y parámetros físico químicos).

La forma general del modelo que se consideró está dada por:

$$
Y_{i j k}=X \beta+b_{i j}+\varepsilon_{i j k}
$$

donde $Y_{i j k}$ es la variable de respuesta (concentración de OD), para $i$ : localización, $j$ : profundidad, y $k$ : campaña de muestreo, $X$ se refiere a las variables conocidas (localización, profundidad, hora, condiciones climáticas y parámetros físico químicos y biológicos), $\beta$ es el parámetro del modelo a ser estimado, $b_{i j}$ el efecto aleatorio que toma en consideración la variabilidad entre la variable de respuesta y las diferentes localizaciones y profundidades, y $\varepsilon_{i j k}$ el error en cada observación.

Mediante (1) se probaron las siguientes hipótesis:

- Diferencia de concentraciones medias de OD entre localizaciones o puntos de muestreo $(\mu 1=$ $\mu 2=\mu 3=\mu 7=\mu 8=\mu 9=\mu 13=\mu 14=\mu 15$ vs. al menos un par es diferente: $\mu 1 \neq \mu 2 \neq \mu 3 \neq \mu 7$ $\neq \mu 8 \neq \mu 9 \neq \mu 13 \neq \mu 14 \neq \mu 15)$.

- Diferencia de concentraciones medias de OD entre las profundidades ( $\mu$ fondo $=\mu$ superficie vs. $\mu$ fondo $\neq \mu$ superficie).

- $\quad$ Diferencia de concentraciones medias de OD entre filas de muestreo $(\mu \mathrm{R} 1=\mu \mathrm{R} 2 ; \mu \mathrm{R} 1=\mu \mathrm{R} 3$; $\mu \mathrm{R} 2=\mu \mathrm{R} 3$ vs. $\mu \mathrm{R} 1 \neq \mu \mathrm{R} 2 ; \mu \mathrm{R} 1 \neq \mu \mathrm{R} 3 ; \mu \mathrm{R} 2 \neq \mu \mathrm{R} 3$ ).

- Diferencia de concentraciones medias de OD entre columnas de muestreo $(\mu \mathrm{C} 1=\mu \mathrm{C} 2 ; \mu \mathrm{C} 1=$ $\mu \mathrm{C} 3 ; \mu \mathrm{C} 2=\mu \mathrm{C} 3$ vs. $\mu \mathrm{C} 1 \neq \mu \mathrm{C} 2 ; \mu \mathrm{C} 1 \neq \mu \mathrm{C} 3 ; \mu \mathrm{C} 2 \neq \mu \mathrm{C} 3)$.

- Diferencia de concentraciones medias de OD en el tiempo $(\mu \mathrm{T} 1=\mu \mathrm{T} 2=\mu \mathrm{T} 3$ vs. $\mu \mathrm{T} 1 \neq \mu \mathrm{T} 2 \neq$ $\mu \mathrm{T} 3)$.

- Influencia de la hora del día.

- Influencia de las condiciones fisicoquímicas y biológicas.

- Influencia de los factores meteorológicos.

Los datos tomados de las diferentes campañas de muestreo se usaron para estimar los parámetros $\beta$ del modelo, los cuales se calcularon usando la máxima verosimilitud restringida (REML) con Proc MIXED en el software estadístico SAS 9.3. F-test se usó para probar la significancia de los coeficientes del modelo.

Finalmente el modelo se comprobó para la convergencia y, cuando la varianza del "random intercept" fue cero, el modelo se reajustó omitiendo el término de efecto aleatorio.

\section{RESULTADOS Y DISCUSIÓN}

Varios factores influencian los procesos de producción y consumo de OD en lagunas de estabilización para aguas residuales, tales como la luz solar, $\mathrm{pH}$, temperatura y concentración de algas (Kayombo et al., 1999). Entonces se espera que los resultados obtenidos en la distribución de OD en las lagunas de maduración se encuentren afectados, hasta cierto punto, por estos factores.

La Figura 3 muestra las concentraciones medias de OD y las barras de error para cada punto con el valor de la desviación estándar como una función de la localización dentro de las lagunas y a diferentes profundidades. Se observa claramente la estratificación de OD, con concentraciones mayores en la capa superficial comparadas con la capa cercana al fondo. 


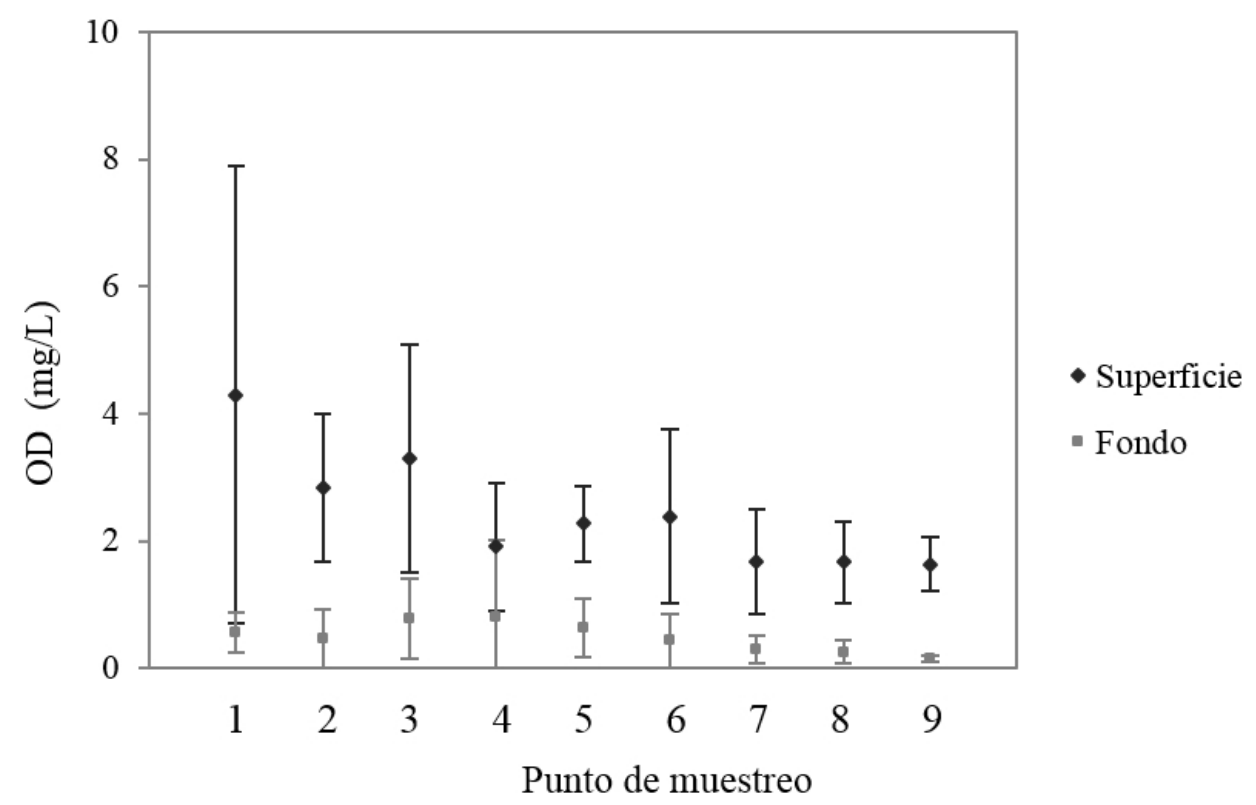

(a)

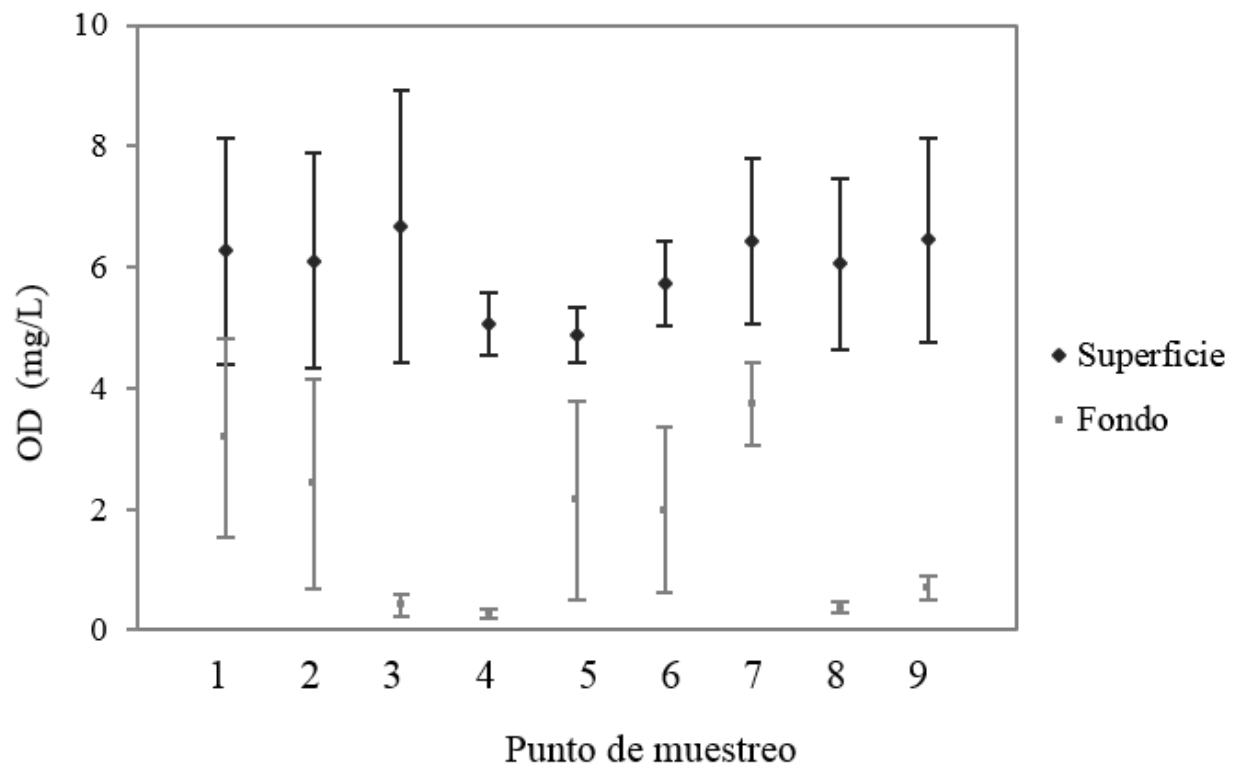

(b)

Figura 3. Concentraciones medias de OD $\left(\mathrm{mg} \mathrm{L}^{-1}\right)$ para todos los puntos de muestreo a dos profundidades (a) M1 y (b) M2.

Además, los $p$ values obtenidos en el análisis estadístico, tanto para M1 $(<0.001)$ como para M2 $(<0.0001)$, confirman el efecto de la profundidad sobre el OD. Este hecho era esperado ya que existe mayor disponibilidad de luz cerca de la superficie.

En cuanto a la influencia de la hora del día, se puede observar en la Figura 4 que los puntos se encuentran algo dispersos a lo largo de las horas del día. Existe una cierta tendencia de mayores concentraciones cerca del mediodía, y en general los valores de T3, son menores a los valores de T1 y T2. En Cuenca (Ecuador), existe luz solar desde las $6 \mathrm{~h} 00$ hasta las 18h00, aproximadamente, con picos de intensidad de luz entre las $13 \mathrm{~h} 00$ y 15h00, como ocurre en los trópicos (Tadesse, Green, \& Puhakka, 2004), por lo que la máxima actividad fotosintética del fitoplancton que produce oxígeno y consume $\mathrm{CO}_{2}$ disuelto se da durante estas horas (Tanner, Craggs, Sukias, \& Park, 2005), como fue efectivamente observado. 


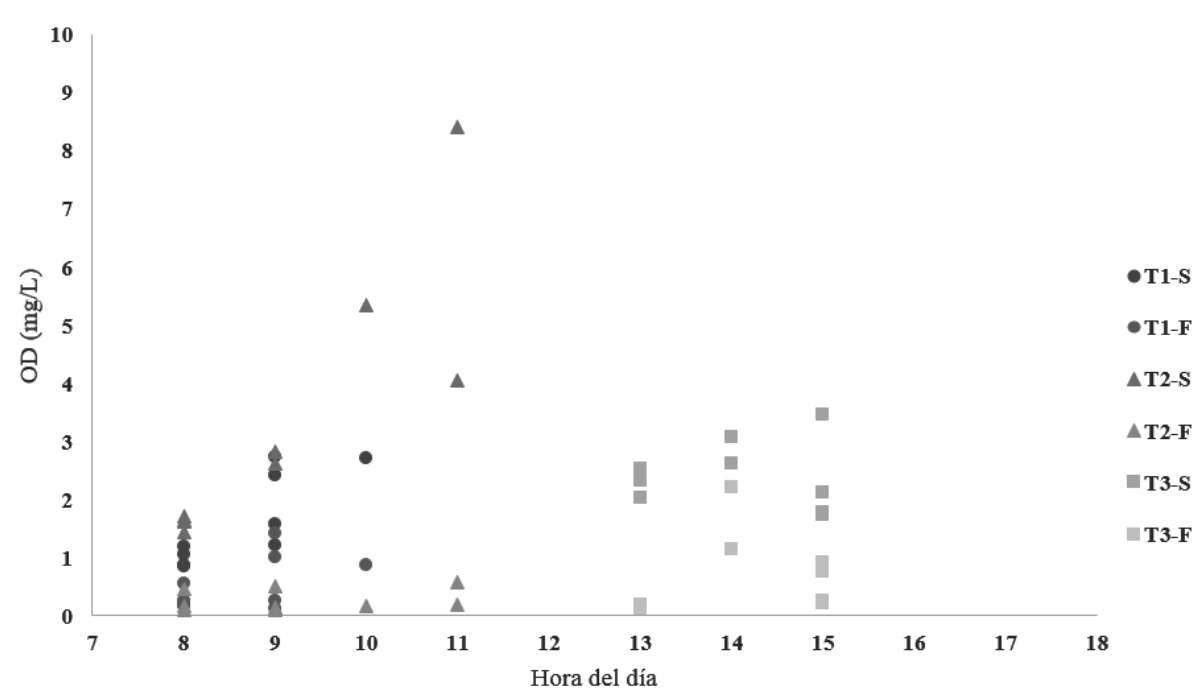

(a)

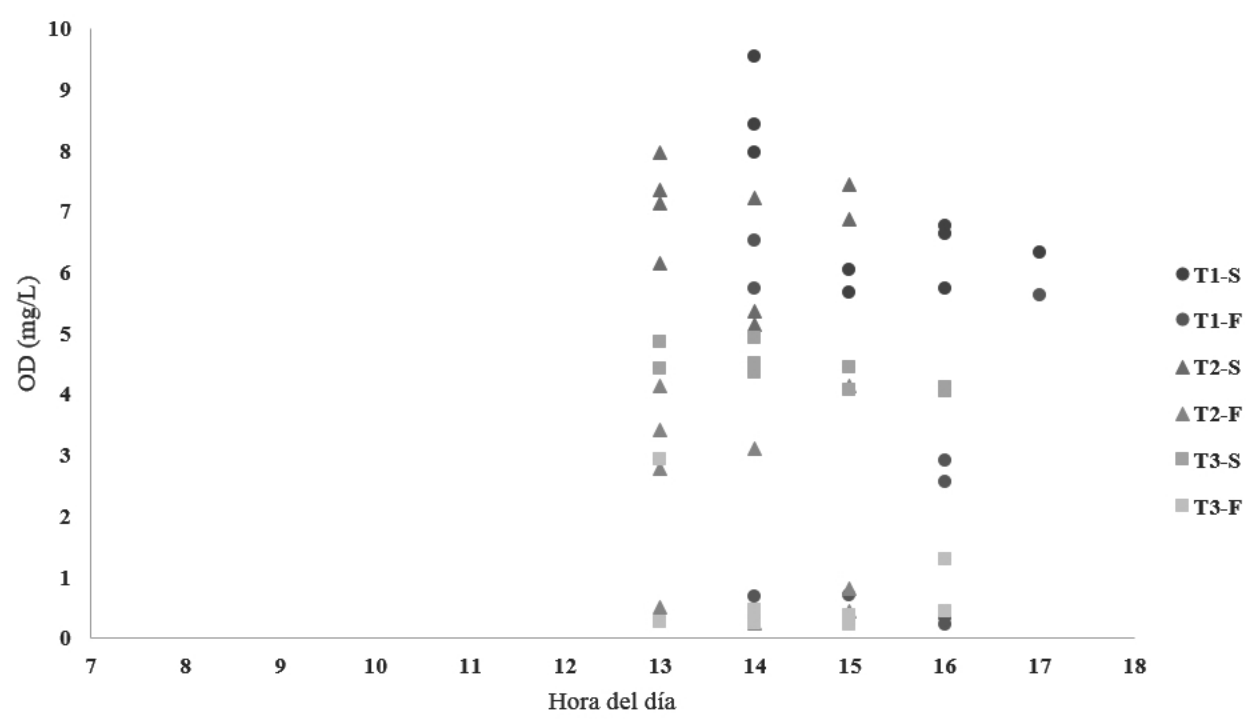

(b)

Figura 4. Relación entre concentraciones de OD (mg L-1) y la hora del día a dos profundidades en (a) M1 y (b) M2. (T1, T2 y T3: primera, segunda y tercera campaña de muestreo; S, F: superficie y fondo respectivamente).

Además, existe diferencia de concentraciones de OD entre las líneas (M1 y M2), lo que sugiere que pueden existir otros factores, que no se han tomado en cuenta, para que se dé este hecho. Abbas, Nasr, \& Seif (2006) estudiaron la geometría de lagunas de estabilización para calidad de agua, y encontraron que al aumentar la relación largo - ancho, la concentración de OD en el efluente disminuía. En este caso, la relación largo/ancho para M1 es de alrededor de 2, mientras que para M2 es de alrededor 1.2. Aunque las dos lagunas de maduración fueron diseñadas de tal manera que trataran el agua hasta el mismo nivel, los resultados obtenidos demuestran que el efluente de la maduración 2 tiene mejor calidad en términos de OD. Se debe poner atención en la división del caudal al ingreso de la planta, ya que al no proveer de la misma carga hidráulica a las dos líneas de tratamiento, la calidad del efluente se puede ver afectada.

En los análisis de contrastes realizados entre filas y columnas (Fig. 2), se encontró que las únicas diferencias significativas se dan en la capa superficial (Tabla 1). Esta capa se encuentra gobernada por la radiación solar durante el día, reflejado en el OD, mientras que en el fondo no existe variación, como ha demostrado Mara (2004) para lagunas facultativas, y Tanner et al. (2005), para lagunas de maduración. Este hecho se replica en las lagunas de maduración de este estudio. 
Tabla 1. Contrastes entre filas y columnas para M1 y M2 (S: superficie, F: fondo).

\begin{tabular}{cc}
\hline Etiqueta & $\operatorname{Pr}>|t|$ \\
\hline S: col 1: M1-M2 & 0.0035 \\
S: col 2: M1-M2 & 0.0049 \\
S: col 3: M1-M2 & 0.0032 \\
F: col 1: M1-M2 & 0.7345 \\
F: col 2: M1-M2 & 0.8657 \\
F: col 3: M1-M2 & 0.7469 \\
S: fila1: M1-M2 & 0.0038 \\
S: fila2: M1-M2 & 0.0038 \\
S: fila2: M1-M2 & 0.0037 \\
F: fila1: M1-M2 & 0.6616 \\
F: fila2: M1-M2 & 0.7812 \\
F: fila3: M1-M2 & 0.9093
\end{tabular}

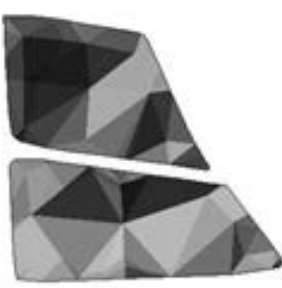

T1

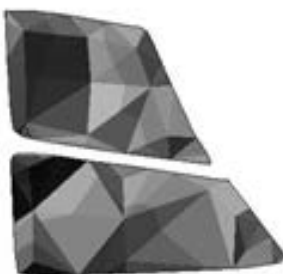

T2

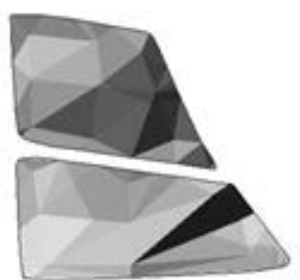

T3

CLOROFILA (ug/)

(a)

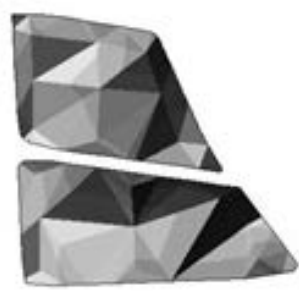

$\mathrm{T} 1$

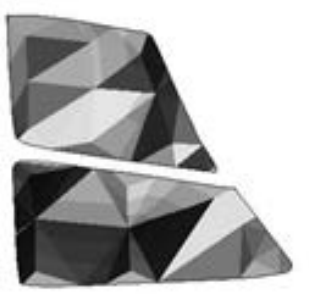

T2

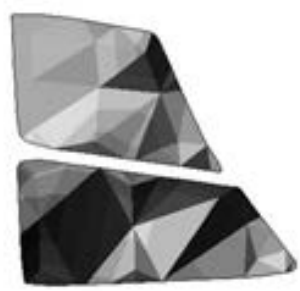

T3

CLOROFILA (ag/)

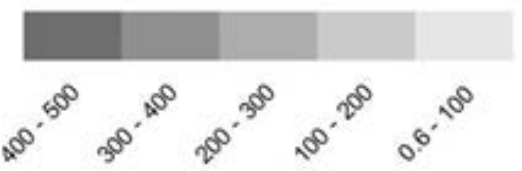

(b)

Figura 5. Contornos de concentraciones de clorofila a ( $\mu \mathrm{g} / \mathrm{L}$ ) en M1 y M2 (a) en la superficie, (b) en el fondo.

De acuerdo a Pearson (2005), la actividad fotosintética de las algas es la responsable de al menos el $80 \%$ de la producción de OD en lagunas de estabilización. Consecuentemente, la distribución de clorofila $a$ (concentración de la biomasa de algas) debería seguir un patrón similar a la distribución de concentraciones de OD, aunque más constante ya que las algas no se reproducen tan rápido. Según la Figura 5, el patrón predominante es que las concentraciones de clorofila $a$ son mayores cerca de la superficie, mostrando una correlación positiva con el OD, lo que además tiene relación con la influencia de la hora del día, como fue discutido anteriormente. 
Los resultados obtenidos para los efectos de los parámetros fisicoquímicos en el OD son bastante diversos (Tabla 2), en el sentido de que ciertos parámetros son significantes para las dos lagunas y otros muestran efectos solo para una de ellas.

De acuerdo a Tadesse et al. (2004) la oxigenación fotosintética (altos niveles de OD y aumento de $\mathrm{pH}$ ) es mayor cuando la radiación solar es mayor. Por otro lado, valores de $\mathrm{pH}$ sobre 9.5 promueven una significante precipitación de P (Pearson, 2005), lo que sugiere que éste debe estar relacionado con el OD. En este trabajo se ha demostrado el efecto del pH sobre el OD, únicamente para M1, pero se observa que P sí presenta un efecto según la hora del día en el OD para M1 y M2. Se podría entonces presumir que altas concentraciones de OD en lagunas de maduración indican precipitación de P. De acuerdo a nuestro conocimiento, no existe mucha investigación sobre el rol que juega el OD en la remoción de P, por lo que es recomendable un estudio más a fondo sobre este tema, sabiendo que, de acuerdo a los resultados de esta investigación, podría existir una potencial relación entre las concentraciones de P y OD.

Los nitratos, al igual que $\mathrm{pH}$, únicamente mostraron tener un efecto para $\mathrm{M} 1$, mientras que en los resultados para el análisis estadístico de Nitrógeno Kjedahl, y Amonio, ninguno presentó efecto sobre el OD.

Tabla 2. Resumen de $p$ values significativos para parámetros químicos sobre OD.

\begin{tabular}{ccccc}
\hline \multirow{2}{*}{ Parámetro } & \multirow{2}{*}{ Laguna } & \multicolumn{3}{c}{$p$ value } \\
\cline { 3 - 5 } & & General & *Prof & *Hora \\
\hline \multirow{2}{*}{ pH } & M1 & 0.0325 & 0.0011 & 0.0058 \\
& M2 & & & - \\
\multirow{2}{*}{ Nitrógeno (N) } & M1 & & & 0.0077 \\
\multirow{2}{*}{ Fósforo (P) } & M2 & & & - \\
& M1 & & & 0.05 \\
& M2 & & & 0.05 \\
\hline
\end{tabular}

Para las condiciones climáticas no se obtuvo ningún resultado significativo, probablemente porque no se tenía ninguna situación de referencia previamente establecida. Así que, los factores meteorológicos podrían haber tenido un efecto en el OD pero no se probaron con una condición de referencia que haga la diferencia.

\section{CONCLUSIONES}

De acuerdo a los análisis realizados en este estudio, los factores que presentaron influencia significativa sobre las concentraciones de OD en lagunas de maduración son la profundidad, hora del día, clorofila $a$ y algunos parámetros químicos.

Se encontró que el OD es fuertemente dependiente de la profundidad, con valores mayores cerca de la superficie del agua. De la misma forma, se evidenció que la hora del día afecta las concentraciones de OD, con los valores pico durante la temprana tarde (entre $13 \mathrm{~h} 00$ y $15 \mathrm{~h} 00$ ), asociado con la actividad fotosintética. Por otra parte, contrariamente a lo que se esperaba, las concentraciones de OD presentaron diferencias según la línea de tratamiento (M1 y M2), lo que indica que la geometría de las lagunas afecta dichas concentraciones. Además, la clorofila $a$ mostró una correlación positiva con la producción de OD, siguiendo tendencias similares de mayores concentraciones cerca de la superficie.

De acuerdo a las pruebas estadísticas desarrolladas, las concentraciones de $\mathrm{P}$ presente en las lagunas de maduración, dependiendo de la hora del día, tiene un efecto significativo sobre el OD. Este hallazgo indica que $\mathrm{P}$ precipita debido a los valores altos de $\mathrm{pH}$ y OD, por lo que se recomienda una investigación más profunda para encontrar las relaciones entre dichos componentes que influyen en la precipitación. 


\section{REFERENCIAS}

Alvarado, A. (2013). Advanced dynamic modelling of wastewater treatment ponds. Doctoral dissertation, Ghent University, Belgium.

Abbas, H., Nasr, R., Seif, H. (2006). Study of waste stabilization pond geometry for the wastewater treatment efficiency. Ecological Engineering, 28(1), 25-34. https://doi.org/10.1016/j.ecoleng.2006.03.008

Adèr, H. J., Mellenbergh, G. J., Hand, D. J. (2008). Advising on research methods: a consultant's companion. Rosmalen, The Netherlands: Johannes van Kessel Publishing.

APHA. (2005). Standard Methods for the Examination of Water and Wastewater. (21 $\left.{ }^{\text {st }} \mathrm{ed}.\right)$. Washington DC.

Dias, D. F. C., Passos, R. G., Von Sperling, M. (2017). A review of bacterial indicator disinfection mechanisms in waste stabilisation ponds. Reviews in Environmental Science and Bio/Technology. https://doi.org/10.1007/s11157-017-9433-2

Kayombo, S., Mbwette, T. S. A., Mayo, A. W., Jorgensen, S. E. (1999). Modeling of diurnal variation of dissolved oxygen in waste stabilization ponds. Ecological Modeling, 12, 21-31.

Liu, S., Ren, H., Shen, L., Lou, L., Tian, G., Zheng, P., Hu, B. (2015). pH levels drive bacterial community structure in sediments of the Qiantang River as determined by 454 pyrosequencing. Front Microbiology, 6(285), 1-7.

Mahmood, Q., Pervez, A., Zeb, B. S., Zaffar, H., Yaqoob, H., Waseem, M., Zahidullah, Afsheen, S. (2013). Natural treatment systems as sustainable ecotechnologies for the developing countries. BioMed Research International, 2013, 796373. Retrieved from http://www.pubmedcentral.nih.gov/articlerender.fcgi?artid=3708409\&tool=pmcentrez\&rendertyp $\mathrm{e}=\mathrm{abstract}$

Mara, D. (2004). Domestic wastewater treatment in developing countries. London, UK: Earthscan.

Mburu, N., Tebitendwa, S. M., van Bruggen, J. J. A., Rousseau, D. P. L., Lens, P. N. L. (2013). Performance comparison and economics analysis of waste stabilization ponds and horizontal subsurface flow constructed wetlands treating domestic wastewater: A case study of the Juja sewage treatment works. Journal of Environmental Management, 128, 220-225. https://doi.org/10.1016/j.jenvman.2013.05.031

Pearson, H. (2005). Microbiology of waste stabilization ponds. In: Shilton, A. (Ed.). Pond treatment technology. London, UK: IWA Publishing, pp. 14-43.

Rivas, A., Barceló-Quintal, I., Moeller, G. E. (2011). Pollutant removal in a multi-stage municipal wastewater treatment system comprised of constructed wetlands and a maturation pond, in a temperate climate. Water Science and Technology, 64(4), 980-987. https://doi.org/10.2166/wst.2011.731

Tadesse, I., Green, F. B., Puhakka, J. (2004). Seasonal and diurnal variations of temperature, pH and dissolved oxygen in advanced integrated wastewater pond system treating tannery effluent. Water Research, 38(3), 645-654. https://doi.org/10.1016/j.watres.2003.10.006

Tanner, C. C., Craggs, R. J., Sukias, J. P. S., Park, J. B. K. (2005). Comparison of maturation ponds and constructed wetlands as the final stage of an advanced pond system. Water Science and Technology, 51(12), 307-314.

Von Sperling, M. (2007). Waste stabilisation ponds. $3^{\text {rd }}$ volume in the series Biological Wastewater Treatment. London, UK: IWA Publishing, 168 pp.

Walmsley, N., Shilton, A. (2005). Solids and organics. In: Shilton, A. (Ed.). Pond treatment technology. London, UK: IWA Publishing, pp. 66-76. 